\title{
Creative Learning with Serious Games
}

\author{
doi:10.3991/ijet.v5s3.1495 \\ Aristidis Protopsaltis ${ }^{1}$, Lucia Pannese ${ }^{2}$, Sonia Hetzner ${ }^{3}$, Dimitra Pappa ${ }^{4}$, Sara de Freitas ${ }^{1}$ \\ ${ }^{1}$ Coventry University, Coventry, United Kingdom, ${ }^{2}$ Imaginary srl, Italy, \\ ${ }^{3}$ Friedrich-Alexander-Universität Erlangen-Nürnberg, Germany, ${ }^{4}$ NCSR Demokritos, Greece.
}

\begin{abstract}
This paper, summarises the Creative Learning with Serious Games workshop that took place in the Fun and Games 2010 conference. The workshop discussed innovative methodological approaches to Serious Games for creative learning. A special emphasis was given to state-ofthe-art research work and cross-discipline approaches (e.g. the mix of Storytelling and Serious Games). In addition, different case studies coming from very different European (research) projects were presented and discussed with the participants. Five papers were selected via a peer reviewed process to be presented at the workshop. The authors presented their work and demonstrated their applications during the second part of the workshop.
\end{abstract}

Index Terms-Serious Games, Creative Learning, Experiential Learning, Storytelling, Game-Based Learning

\section{INTRODUCTION}

Serious Games (SG) are entering more and more the educational world. Games in education make it possible to overcome practical constraints of real-world settings and explore the far greater boundaries of virtual spaces. They promote creative and self regulated learning either within the classroom or at home. Many approaches to creativity stress human potential to self-realization, personal growth and fulfilment, openness and flexibility [1] and Serious Games seem to fit this account very well.

Modern Game-Based Learning (GBL) approaches are commonly build on applications that have defined learning outcomes and are designed to promote active participation and interaction, balancing the learning objective with the gameplay, in order to enhance the ability of the learner to retain and apply the knowledge gained to the real world while being completely involved and thus more receptive. SGs often exploit narratives, storylines, visual elements and other features common to entertainment games, such as scoring and social networking in order to motivate and engage players in a learning activity. Educational games have learning goals and structure, but in addition are adaptive and interactive and most importantly they provide enjoyment, pleasure, motivation, gratification and emotion, in order to achieve learner engagement and involvement.

Games create simulated environments that facilitate immersion, allowing learners to explore alternative approaches to situations virtually, in order to directly experience practical and emotional consequences of their actions. Through these approaches, GBL allows learners to directly though virtually experience the real-world by developing their awareness of real situations. For example, SGs can combine a wide range of different kinds of inputs and outputs for understanding complex social issues like immigration, cultural differences, social integration, etc.
This requires adopting multiple viewpoints and scenarios to analyse cause and effect. SGs provide a safe environment for active, critical and creative learning, allowing users to explore skills, methods, and concepts. The potential learning outcomes include changes in participants' behaviour, knowledge, skills, attitudes, and/or levels of functioning.

In the last few years due to the widespread use of commercial games the domain of Games-Based Learning has received increasing attention and the potential of Serious Games in learning and training has been started to get wider recognition. However, until recently strategies for supporting the more effective methods of learning with games were uncertain [2]. Research within the Engaging Training Solutions project [3], has found that a comparison of traditional learning and Game-Based Learning yielded significant difference in favour of Game-Based Learning. Other studies have also confirmed this finding [e.g. 4].

Some of the main strengths of Game-Based Learning include motivating learners and providing personalised learning approaches for either individual users or groups. Motivation is a key aspect of effective learning, but such motivation needs to be sustained through assistive methods such as feedback responses, reflection and active involvement, in order for designed learning to take place [3]. Additionally, GBL offers the potential to integrate different cognitive tools that scaffold important processes of reflection and articulation, such as discussion forums, bulletin boards and concept mapping software and this is particularly evident in multiplayer online games, where users can use other software tools and achieve collaborative learning. Nevertheless, it is important that learning is congruent to lifestyle of the learner and in the case of Serious Games it should be congruent to the player's lifestyle for effective learning to take place [5].

Computer games can help players to acquire or improve certain cognitive abilities and skills such as eye-hand coordination, visual attention, spatial abilities, visual acuity, ability to simultaneously track multiple moving visual items, multisensory processing abilities, proactive thinking, systematic organisation of information, means-ends analysis [6] and improve understanding in topics [7-9]. Players have to manage multiple inputs and objectives simultaneously, different resources, and to make instant decisions. Furthermore, often, people who play such video games exhibit increased fluid intelligence [10] which is associated with problem solving abilities [11] and consequently supports creative learning. Serious Games provide an environment for active, critical, often collaborative and creative learning, allowing users to explore methods, concepts and skills rapidly and safely within an environment designed with specific learning components. 
From a pedagogy perspective well designed Serious Games have different aspects of different learning theories integrated in the design. These learning or pedagogic approaches include Problem-Based Learning (PBL), as well as contextual and experiential learning models. The eVITA project [12] is one example of such approach. The project is aiming to provide knowledge about the cultural differences in Europe and test different learning approaches. Life-experiences collected from the narratives of older citizens (Storytelling) are transposed to Serious Games. Each game has the same learner, context, and representational medium, yet the pedagogic foundations are varied so as to provide a basis for comparative study.

The four approaches include:

1. A narrative-based game which uses storytelling to achieve engagement and flow;

2. An experiential game, influenced by situative pedagogy where the player is transferred into the state of affairs faced by the narrator;

3. A puzzle-based game, where the player has to apply problem solving skills to overcome challenges and solve puzzles, and finally;

4. An exploratory game focused on increasing the learner's zone of proximal development, by using external material and resources in order to overcome the challenges presented by the game.

By combining these methodological approaches, the eVITA project promotes and investigates pedagogy driven innovation. Therefore, a set of 'European cultural games' about cross-border experiences was developed, allowing users to experience Europe before the EU, when strict travelling and migration rules, different economic and monetary systems was the norm.

Investigating creative learning with SGs, brought together innovative methodologies to SGs and case studies of real-life applications of GBL, which, after being briefly introduced, were be intensively discussed. The workshop was structured in two parts: the first part featured the presentation of the e-VITA project along with peer reviewed relevant paper presentations and the second, short demonstrations of innovative GBL solutions (Interactive Exhibition). A SG is a game in which learning is the primary goal. A careful pedagogic and instructional design is required to create games to provide 'serious' experiences for different application areas, always taking into consideration the expectations of the intended players. Another critical aspect of SGs development is the definition of story board and graphical design elements, in line with the selected pedagogical approach and the corresponding workflow. Achieving a balance between educational approach and game elements is essential to the success of GBL solutions. In this light, the following topics were addressed during the workshop:

- Pedagogical approaches for Serious Games

- Development of Serious Games Storyboards

- Case studies of creative learning with SGs

\section{THIS IsSUE}

The five papers selected to be part of this special issue about creative learning with Serious Games (SG) for learning are following briefly described.

\section{A. Emotions in Serious Games: From experience to assessment}

Serious Games (SG) offer different benefits due to the integration of simulation aspects, game aspects and pedagogical elements at the same time. Among the different assets that SG provide for learning, this paper focuses on their ability to leverage the synergy between emotional and learning appropriation processes. First, the specific features of learning opportunities offered by Serious Games considering the involvement of emotions in this learning process are outlined. The following part of the paper is dedicated to the description of the methods for the analysis of the emotional experience of SG users. Finally, the paper explores a new promising research direction concerning the use of Affective Computing in Serious Games.

\section{B. Towards a framework for learning in the OSMA Serious game engine}

Online multiplayer serious games offer a way to support learning in a gaming paradigm that is familiar to many players and has proven its effectiveness in providing sustainably enjoyable gameplay. The aim of this paper was to decrease development cost for these games by providing a modular game design framework and a component-based technical architecture. The technical architecture and the game design framework was implemented and iteratively refined through two proofs of concept.

\section{The character of successful trainings with serious games}

This paper gives a basic guideline for the development of trainings using serious games. It describes a framework for the training with Serious Games. It shows how to define a training goal and describes the process of learning to reach this goal. It points out a methodology how to design successful trainings with Serious Games.

\section{The use of competition and creativity as key driver to promote scientific culture among students}

The use of didactic games in order to promote scientific culture leads to innovative methodologies and technologies that can be very effective for the learning process of students or simply to make them more curious about "critical disciplines" such as mathematics. Delivering learning games means making an effort to meet the language and the culture of learners, focusing on their motivation: this aim can be achieved by developing projects and products with a high use of competitiveness and creativity.

\section{E. Designing Effective Serious Games. \\ Opportunities and Challenges for Research}

The final paper has investigated the state of the art research on SGs, starting from the cognitive aspects that are necessary in order to root technological development and applications in sound theoretical foundations. The paper discusses some key aspects of SG design and exploitation: choice of components-off-the-shelf or from-scratch design, tools and methodologies for development or adaptation, intelligent tutoring, virtual coaches and affective learning, living worlds, game mechanics, HumanComputer Interaction. The paper proposes several research questions - that range from requirements elicitation 
to design and from deployment to use and evaluation - to be answered in order to avoid technology pushing and drive technological research according to the requirements of the end-users and stakeholders. Finally, the researchers propose four major directions that can strengthen the foundations of SG research: definition of metrics and learning progress evaluation tools; methodologies and tools for designing games from various topics and for various users; computing and communication architectures; technologies that can enhance the overall system performance.

\section{CONCLUSION}

Learning is often described as an active process, in which meaning is developed on the basis of experience. The work presented during this workshop has demonstrated the applicability of educational games for fostering learning and motivation in complex learning situations, as well as the complexity and challenges surrounding the design of effective didactic games. Overall, SGs design calls for a holistic examination of a number of parameters that relate for example to the pedagogical objective(s), the indented target group(s), the educational environment etc.

Consequently, given that SGs can be extremely diverse in terms of audience, specific learning goals, topic etc. the biggest challenge for future research in the field is probably to collect evidence from diverse game and contextspecific observations to systematically contribute to the development of a generic theory for GBL.

\section{REFERENCES}

[1] Treffinger, J.D., G.S. Isanksen, and L.R. Firestien, "Theoretical Perspectives on Creative Learning and it Facilitation: An Overview", The Journal of Creative Behavior, 17 (1 First Quarter): p. 9-17, 1982.

[2] de Freitas, S. and M. Oliver, "How can exploratory learning with games and simulations within the curriculum be most effectively evaluated?" Computers and Education, 46 (3): p. 249-264, 2006. doi:10.1016/j.compedu.2005.11.007

[3] Jarvis, S. and S. de Freitas, "Evaluation of an Immersive Learning Programme to support Triage Training. " in 1st IEEE International Conference in Games and Virtual Worlds for Serious Applications, Coventry, UK: IEEE Computer Society, 2009, 117-122.

[4] Mautone, T., V. Spiker, and D. Karp, "Using Serious Game Technology to Improve Aircrew Training" in Interservice/Industry Training, Simulation \& Education Conference (I/ITSEC), Orlando, FL, 2008.

[5] Pappa, D., et al., "Game-based learning for knowledge sharing and transfer: the e-VITA approach for intergenerational learning", in Handbook of Research on Improving Learning and Motivation through Educational Games: Multidisciplinary Approaches, P. Felicia, Editor, IGI Global, In Press.

[6] Vorderer, P. and B. Jennings, "Playing video games: Motives, Responses and Consequences", Mahwah NJ [u.a.]: Lawrence Erlbaum Assoc., 2010.

[7] Becta, "Computer Games in Education", Becta: Coventry, 2006.

[8] Aguilera, M. and A. Mendiz, "Video games and education: education in the face of a "paralel school"', Computers in Entertainment, 1 (1): p. Article 8, 2003.

[9] Jenkins, H., et al., "Entering the Education Arcade", Computers in Entertainment, 1 (1): p. 17-28, 2003. doi:10.1145/950566.950591

[10] Perez, R., "Researchers Examine Video Gaming’s Benefits", D.o. Defence, Editor, 2010.
[11] Cattell, R.B., "Intelligence: Its structure, growth, and action", New York: Elsevier Science Pub. Co, 1987.

[12] e-VITA, The e-VITA project is funded under the LLP-KA3 programme : www.evitaproject.eu.

\section{AUTHORS}

Aristidis Protopsaltis, Serious Games Institute at Coventry University. His research interests lie in the area of Serious Games, Information Processing, Human, and Computer Interaction. Aristidis has spent several years as a visiting lecturer and researcher at various universities in the UK. He is managing three European projects and has participated in several other projects. He has published a number of peer-reviewed conference and journal papers. He is currently the vice chair of the European Chapter of ACM SIGDOC.

Lucia Pannese, imaginary srl, graduated in Mathematics, has managing experience in (research) projects with special attention to technology enhanced learning solutions. In 2004 founding partner of imaginary s.r.l. a company designing and developing Serious Games and simulation systems; at present covering the position of CEO. In 2008 she founds Games2Growth Ltd based at the Serious Games Institute, Coventry University Technology Park. Lucia is the coordinator of the e-VITA project.

Sonia Hetzner, Institute for Innovation in Learning (FIM New Learning), University of Erlangen-Nuremberg, Germany. Sónia Hetzner is a Senior Researcher. Currently she is leading the research area "Game-based Learning and Serious Games" at the Innovation in Learning Institute. She is social scientist, geographer and pedagogy expert. She has been responsible for the conceptualization, development, management implementation and evaluation of technology-enhanced learning and training environments for different target groups. Sonia Hetzner has long-term experience in European and national projects as project coordinator and evaluator.

Dimitra Pappa, National Centre for Scientific Research"Demokritos”, holds a degree in Electrical Engineering from the National Technical University of Athens Greece (NTUA), specialising in telecommunication technologies. She has taken part in a number of national and European research and development projects in the fields of eLearning, telemedicine, eGovernment and eCommerce. She has published several papers in journals and international conferences.

Sara de Freitas, Director of Research at the Serious Games Institute, Professor of Virtual Environments and Fellow of the Royal Society of Arts. Sara leads the applied research team at the Serious Games Institute and the Serious Games and Virtual Worlds Applied Research Group at Coventry University.

This paper is an extended version of a presentation given during the "Creative Learning with Serious Games" workshop during the "Fun and Games 2010" conference in Leuwen on 15 September 2010, and was supported by the e-VITA European Life Experiences", project $\mathrm{nr}$ 143526-LLP-1-2008-1-IT-KA3-KA3MP.

Submitted October $30^{\text {th }}$, 2010. Published as resubmitted by the authors November $11^{\text {th }}, 2010$. 